\title{
Features, Enhancements and Perspectives of State Estimation in Serbian TSMO
}

\author{
Jelena R.Veselinović ${ }^{1}$, Miloš V. Stojić ${ }^{2}$ Goran S. Jakupović
}

\begin{abstract}
Serbian transmission and market system operator has two operational SCADA/EMS systems working in parallel. During the years of working in production, some weak points in applications functioning have emerged and they have triggered improvements in the process of planning and specifying technical requirements. In the process of upgrade of these systems, new important features have been carefully tested through many additional user-designed (unstructured) tests, giving a new set of ideas for further improvements. In this paper, we will look back at some of the most frequent problems emerging in continuous real-time state estimation operation and their correlation with our improvements. We will also point out some other interesting features related to SCADA/EMS, as seen through the eyes of a SCADA/EMS engineer and Power Systems Analysis engineer. Also, the authors will give an overview of some key requirements for more reliable state estimation process and point out other affecting factors, considering its role in market environment and the need for its very stringent percent of availability. A brief overview of current and future work to improve EMS applications in order to meet requirements of future regional market integration will be given at the end of the paper.
\end{abstract}

\footnotetext{
${ }^{1}$ PE Elektromreža Srbije

Serbian Transmission System and Market Operator ICT Operational Technology Department

Vojvode Stepe 412, Belgrade, Serbia (e-mail: jelena.veselinovic@ems.rs)

${ }^{2}$ Mihajlo Pupin Institute, University of Belgrade, Volgina 15, Belgrade, Serbia

(e-mail: milos.stojic@pupin.rs,goran.jakupovic@pupin.rs)
}

Keywords- SCADA, EMS, Network Topology, State Estimation

\section{System Overview}

Serbian TSMO has two SCADA/EMS systems working in parallel. These systems run 24 hours a day, 365 days per year, without any "down time" allowed. The principle of redundancy of hardware and software on both of these systems is respected.

SCADA/EMS system hosts all SCADA/EMS databases, performs SCADA processing and EMS calculations, provides data for historical information system, sends data to operators consoles etc. SCADA/EMS functions are considered as critical.

Main software subsystems are:

- SCADA system

- Power applications system: Network, Generation and Planning

- Dispatcher training simulator (DTS).

Real time State Estimation is the first and the most important application of Power Network Analysis.

\section{State Estimator}

State estimator (SE) was the first EMS application introduced in Serbian Transmission System and Market Operator. The state estimator computes the static state of the system (voltage magnitude and phase angle) by monitoring available measurements. It is a powerful tool, with a primary role of helping dispatchers in cases when they have no data available from some substations. Secondly, it is used as an optimization tool, creating the dataset, which is considered as more accurate and reliable in comparison to the input dataset acquisited by SCADA. The output dataset of state estimation is used as a

This is an Open Access article distributed in accordance with the Creative Commons Attribution Non Commercial (CC-BY-NC-ND 4.0) license, which permits others to copy or share the article, provided original work is properly cited and that this is not done for commercial purposes. Users may not remix, transform, or build upon the material and may not distribute the modified material (http://creativecommons.org/licenses/by-nc/4.0/) 
basecase for all the other real-time EMS applications, real-time contingency analysis and pre-dispatch powerflow. Other than that, its results are used by Dispatcher training simulator, as a realistic starting point for simulations.

Prior the state estimation, network topology processor performs so called pre-estimation analysis by executing various tests. One of the most common tests refers to the most illogical situation in network, when there is an opened switching device, paired with non-zero flow measurements. The other one is the incoherency of voltage bus measurements.

After filtering its subset of data, state estimator runs observability analysis, determining the observable area or areas of the network, introducing so called pseudo measurements, where needed, in order to restore observability. At this point, if a certain level of observability has been reached, the state estimator calculation is performed.

Afterwards, state estimation runs its bad data analysis, with a goal to detect, identify and reject all the bad input data. After this last filtering and analysis, state estimator processes this final input set of data, in order to get the final state estimation results.

\section{Problems}

State estimator applications, produced by different manufacturers, like in Serbian TSMO, have some different characteristics, but still, there are some common crucial factors, affecting strongly the possibility to converge.

To begin with, the most important factor is the topology of the network. State estimator calculations "leans" on the graph of the network, to which all the input state estimator eligible measurements are assigned. One topological node can be in correlation to one or more measurements of the same type (voltage, active power, reactive power, position of the tap) or none. So, without a realistic graph of the network, all the equations inside the state estimation calculation can not be properly set and we consequently "spoil" the solution results. Since the graph of the network is formed based on connections, established by switching equipment status measurements, it turns out that state estimation is very sensitive to errors in network topology. Invalid switching equipment statuses (of circuit breakers and disconnectors) are the main cause. Most critical situations and solutions are to be considered.

Determining factors, which can make things go so wrong, before a state estimator was even "given" a valid topology to set the equations properly is the first step.

The most common problems, seen through the eyes of a SCADA/EMS engineer are:

\section{A. Non-telemetered substations}

No measurements of any kind at all, thus no graph formed based on SCADA status measurements. Dispatchers should manually set at least statuses correctly and update them regularly, based on the information coming from the field. However, experience shows that this is a bad solution, due to human factor and a lack of time during dispatcher's shifts.

\section{B. Telemetry failures in telemetered substations}

They can be caused by telecommunication problems or problems with measuring equipment in the field. Aggravating factor is the unpredictability of problems alike, in terms of starting time and a location (substation), where the problem arose. Once the state estimator consequently stops converging, SCADA/EMS engineer has just been assigned another problem, which frequently overgrows the basic cause of non - convergence. Without estimation reports being updated, there is no much information available. Roughly speaking, we stay in the dark.

\section{Large scale telemetry failures}

More critical than B., very possibly resulting in a loss of convergence and once again we can "stay in the dark". This kind of problems can occur also when having problems with data collected by FEP servers, i.e. data coming from different RTUs, in case of multiple RTU outages (as seen on FEP servers in Serbian TSMO) or in case of very rare problems with ICCP data (Open Access Gateway - OAG).

\section{Problems with servers and their interfaces}

They are very rare and can cause a temporary lack of measurements on an enormous scale. Luckily, these kinds of problems are the quickest ones to be spotted, because they produce a sudden loss of most measurements on SCADA displays, constantly observed by dispatchers.

\section{F. Problems related to database errors}

Database corruption is very rare - also unpredictable and sometimes very hard to be recognized as a cause of the problem, or database mistakes caused by human factor error, worsened by the fact that the human does not suspect at first at this possibility at all. Therefore, the last one is maybe the hardest one to recognize, especially if a change of some value or record globally is something that 
has been done completely unconsciously.

A database can be "technically" valid, but there might be a problem with some data. Actually, we are speaking here in terms of just one value, which can make the calculation data become so incoherent to the processor, that it just crashes and once again, in 99\% without any message on the possible cause.

Corrupted database problem is usually solved by retrieving one of the older versions of the database online and usually after having restarted unsuccessfully SCADA/EMS more than once. SCADA/EMS restart messages might not always let us get the insight of what the cause of the problem is.

Human errors in database can cause the same effect like corrupted database does, or they can lead to process failures of the corresponding applications. Having a log of at least latest database changes can help us detect and solve many problems to a big extent. However, not all editors have this log. For example, there was a case of non-convergence, due to an invalid value of a line parameter-resistance. Although SCADA/EMS engineer updated this value correctly, some action or error has taken place in the meantime, causing the crash of a state estimator.

In situations like these, it is very important to review the latest possible changes on the system and make an inspection.

G.) Data acquisition - statuses and measurements, where data have a valid status quality flag, but the values are wrong

This usually happens on a substation level and the problem is not always easily spotted, because there is no flag BAD on SCADA displays, nor on OAG and FEP servers datasets (Open Access Gateway and FEP servers) and especially when these data have a similar value to some usual values.

\section{H.) Problems in Serbian external zone of observability}

In parts of network model "covering" neighbouring countries. Here, we have small and large scale problems regarding data. The aggravating factor lies in the fact, that those are not Serbian substations. The most common problem here can be caused, for example, by some irregular statuses of switching equipment, and it is very hard to set them manually in this situation.

\section{Analysis}

\subsection{Voltage level vs. topology error}

Topological mistakes caused by wrong statuses have a different influence on state estimation convergence, i.e. their impact on state estimation follows the rule - higher the voltage level - higher the influence on convergence.

This means that it is a priority to set statuses of 400 $\mathrm{kV}$ (the highest rated voltage present in Serbian TSMO) switching equipment and especially in places where there is a transformation from $400 \mathrm{kV}$ to some other voltage level ( $220 \mathrm{kV}, 110 \mathrm{kV}$ ). These mistakes are the worst, and in some cases, can even lead to non-convergence. Then, $220 \mathrm{kV}$ and $110 \mathrm{kV}$ levels statuses and other voltage levels statuses.

\subsection{Redundancy - less can be more}

State estimation idea is grounded on the idea of having redundant measurements. It means that if we have $n$ nodes, it is enough to have a vector of $2 n-1$ input known variables to solve the networks state vector, so we have to provide more than this minimal number of measurements. From the state estimation dataset measurements, it is possible to exclude certain measurements. This, we do to avoid their influence to state estimation calculation, in cases when we know that they are wrong, from whatever the reason is.

Having bigger number of measurements is desirable and generally considered as positive. It is true, especially in cases of a lower observability level and a need to increase it. However, by increasing a level of redundancy in areas of network, already "covered" with measurements and provided with minimum of telemetry, more possible mistakes in our state-estimation calculation are also introduced. This is why it is important to find a good measure in the process of adding new available measurements.

\subsection{Taps}

Measurements of tap positions on regulated transformers can have a significant influence on state estimation. On one of SCADA/EMS systems in Serbian TSMO, big reactive energy incoherencies (mismatches) are possibly related to invalid tap measurements. These errors can even lead to a non - convergence, so it is very important to control these measurements and intervene, if necessary. Problems appear especially, when these positions are supposed to be manually updated and they're dependent on the system of informing dispatchers on field updates and whether these updates have been entered on time. 
Tap positions are a common reason for errors in energy management system and they have a significant impact on the state estimation solution quality, which is directly related to all the other network analysis applications.

\subsection{Data availability / processing}

State estimator has a measurement error detection and identification as a part of a state estimation process. For example, if one SCADA substation receives $n$ eligible data that can be input into state estimation application, it means that the number of data that can really be used by state estimator, can be smaller than $\mathrm{n}$. If the quality status of SCADA measurement becomes bad at some moment, according to SCADA processing rules, this measurement will not be used in state estimation. One of the things that can be used as a sign of input data quality and as one of the signs which can point out the reason of state estimator non-convergence is the data availability. Data availability should show the percentage of available data inside the subset of state estimator input data.

\subsection{The model, Load model}

State estimator uses a network model (equations and parameters), available measurements from SCADA and pseudo measurements, which can be driven from load and generation bus injection models, in general.

All the parameters comprised within the model can influence and introduce errors in the process of state estimation. Load model has parameters, describing the loads and also factors, describing their participation in the load of an area.

State estimator uses, by default, measured values active and reactive power of the load, in cases where these measurements are available. When the measurement of the load becomes unavailable, state estimator will use a load model value, which is actually a history updated value of that particular measurement, updated along the state estimator previous history runs, and use it as a pseudo measurement.

Load model of a Serbian system-load model uses load schedules which are normally updated starting from the top of the load model (load area level) downwards. Top level load model value is updated based on the current interchange of Serbian tie-lines and the current sum of Serbian Generation and load- forecast data.

Furthermore, using this top load area value of the modelled load and hourly based load schedules in combination with distribution load factors, uniquely defined for every load in the system, loads are calculated.

Load model of neighbouring systems: non-telemetered top load area values affect the load model of foreign TSOs, introducing accuracy deterioration as well.

\subsection{Network limits}

Network limits of loads and generators have the most important role in model tuning of foreign equivalents, particularly in terminal endings. Network limits (set of Maximum and Minimum values) determine state estimator energy distribution and they have to provide sufficient energy, especially in non-telemetered and terminal points of the model. Transmission Planning and Analysis Department provides these data.

\section{Solutions - improvement features and requirements}

During the years of exploitation, one idea appeared state estimator with the possibility of equivalenting certain parts of the network. We needed some solution that covered problematic situations, described in the previous chapter. This solution had to be simple and applicable down from the level of one bay in the substation hierarchy, up to the level of substation and area. By area, we assume electrical network of one country. As a result of this idea, a new feature was created and implemented as a part of the State estimator (IMP) and its principle will be explained further.

But, first, let's take a look back at the part of state estimator, called Network Topology processor. It has been already mentioned, that the starting point in the process of state estimation is a network topology construction. This is actually done by Network Topology processor, which is building the equivalent network model, using static connectivity of network equipment and values of switching devices input measurements (digital statuses), acquisited by SCADA system. It also detects energized islands.

\subsection{New Feature - Network Model Reduction}

On our proposal, new feature of network model reduction has been implemented in IMP EMS software.

The Network Topology Processor function now allows network model reduction by removing selected part of the network. User selects substations and voltage levels inside for reduction. Also, the whole reduction function can be switched on and off. Such reduced model is presented to all other network applications. This may be useful in situations where telemetry is compromised in confined part of the network. Also the process of network model building or expansion is prone to errors. Debugging by method of elimination is only possible if 
parts of network model may be removed.

Such reduced model is used by all other network applications: State Estimation, Contingency Analysis, Dispatcher Power Flow etc.

Reduction is performed by removing branches having both ends in removed network part. Border branches which have only one terminal in removed part are kept in the model. These terminals are now border buses in the reduced model. These buses require special treatment in network applications.

Branches emanating from these buses must end in non reduced network part. Branches with both terminals connected to border buses are removed from the model. If some power injection measurements exist in such buses, they are removed from measurements set entering state estimation.

Zero virtual injection measurements are not allowed in such buses. For load-flow calculations these buses have PQ type. During load -generation balancing in Dispatcher Power Flow, estimated loads in these buses must be also taken into account.

\subsection{Additional statistics, logs and displays}

We are proposing some additional statistics, logs and displays for implementation in SCADA/EMS systems. These new features are to be implemented in one our SCADA/EMS.

\subsubsection{SCADA data (availability) statistics}

Aside from data availability screening of the input state estimator data set, separate SCADA data screenings by substations are going to be implemented, i.e. SCADA data statistics .

These numbers will be expressed in percentage and shown on SCADA displays as well, and they will be an indication for eventual telemetry problems. They should serve as an alarm for dispatchers and other engineering staff.

\subsubsection{Logs}

Logs - it is very important to keep track of all updates on SCADA/EMS system, on two levels: the first one is having a $\log$ of database updates in modelling environment, and the other one is having a log of updates, made on real time state estimator database and application settings. These features play a significant role in the error debugging process. Online changes, like inclusion or exclusion of a measurement in state estimation, changes of measurement priorities, manually setting of a measurement/status, application settings regarding a state estimation criteria or mode, network reduction of a certain part of a network etc.

\subsubsection{SCADA estimation history display/log}

State estimator should also be equipped with a display, giving a user an insight into the history of state estimation solution and convergence process - information on status and ending time point of a state estimation cycle in real time, all related relevant information, including eventual crashes of a state estimation process on the server itself, the most relevant information on state estimation convergence criteria violations and validation, as well as the information on percentage of valid state estimator input data out of those forwarded to topology processor and state estimator. This display should keep data for a few days.

\subsubsection{SCADA displays - additional SE indications}

One line substation displays - on one line substation diagrams, dispatchers have the ability to replace a SCADA value, with an estimated value, with the possibility of colouring estimated values in accordance to state estimation quality, which can be: not up to date, invalid, valid estimation and not initialized. As an addition to this, a visible indication in case of a reduction of a voltage level or a substation must be present.

\subsection{Robustness}

One of the most important characteristics of any state estimator is its resistance to telemetry errors. In order to test it, a series of unstructured, carefully designed tests, has been performed: for example - simulation of "frozen" measurements, still having a valid (good) quality flag. This situation is very specific and it is important to test it. Simulations were carried out on the example of one bay of a line, then when the problem is spread to the whole $110 \mathrm{kV}$ (for example) level of a substation and finally when the problem exists on the level of the whole substation.

Previous tests were repeated with varied accuracy coefficients, for the simulated measurements, and finally these results were compared to the situation, when these measurements were excluded out of estimation process.

As an example of a remedial action, $110 \mathrm{kV}$ level of this substation was reduced by Network reduction model function and the results were compared once again.

The other tests dealt with sensitivity to other network parameter changes, like line parameters or tap position changes, then very important incoherency tests like a branch flow present with an opened circuit breaker or a zero branch flow combined with an opened circuit breaker etc. These tests also had a purpose of checking different 
online forms of a state estimator.

\subsection{Accuracy classes}

On one of SCADA/EMS, used in Serbian TSMO, there is no possibility of network model reduction, so another solution had to be found for telemetry problems in external observability zone. Problems of this kind are very common.

A special accuracy class for measurements in this external zone was introduced and these measurements were supposed to make a difference in "resolution" between Serbian network and foreign network, inside the state estimator.

However, these changes in cases of big telemetry problems in the external zone did not succeed to compensate these problems, but only alleviated them slightly. As topology errors prevail over measurements, this approach didn't give such good results.

\subsection{Topology vs. measurements}

On our new SCADA/EMS system, in modelling environment (SCADA/EMS database), measurements are independently modelled and defined from network topology.

This facilitates working in the database and especially in periods of maintenance and substation reconstruction, when there is a necessity to "disconnect" measurements from one station bay and "connect" them to another one, or to a bay in different station, depending on a "new" topology scheme, without having to delete them from the database in order to add and attach them to a different device in the process.

It is also possible to make temporary lines, which can be attached temporarily to some already existing measurements, which have been already tested through previous point-to-point test.

Every deletion of a measurement, once tested, is a risk of a possible mistake.

\subsection{SCADA/EMS database timeline}

Newest versions of databases also give a possibility to have individually modelled and saved changes (one or a group of different changes), which can be implemented at a certain point on a timeline in the future.

We don't have this possibility in Serbian TSMO yet, but this would be very important and useful, having in mind, that it takes some time to model SCADA/EMS changes and that the moment at which this change can be put online, can be postponed in the future or undone, which happens very often in reality.

\subsection{Test environment}

A possibility to have a new SCADA/EMS model tested, before putting it online, is very important. This can be realized using a Dispatcher training simulator (DTS) or by validating the model and testing it in a study mode of the state estimator.

Nowadays, worldwide, there is a new feature, which enables a system engineer to load a new SCADA/EMS database in a test - state estimator application, working in parallel with a default real time state estimator application, which is optimal.

\subsection{Modelling outside the responsibility area}

The problem of state estimation convergence: due to neighbouring TSOs telemetry outages, bad data in telemetered subset of data, non telemetered points of the model, inconsistencies in the model.

Making good equivalents for terminal ends and non telemetered parts of the external observability area that need to be taken into account. In a few specific cases for some model inconsistencies, additional loads have been modelled, supported by manually entered pseudomeasurements, which eliminated errors.

\subsection{Mandatory daily check procedures}

A general practice in Serbian TSMO is to perform regular daily SCADA/EMS system health checks. This includes software, hardware and basic network operations checks. Old system is checked manually by performing a set of procedures, defined by manufacturer. For the new system, the set of automated procedures were developed in-house, which relieves system engineers from performing most of the manual checks. By performing these checks, many factors, affecting SCADA/EMS applications, can be prevented or spotted, like process crashes, telemetry outages, hardware problems, etc. Further development of automatic checking procedures would be beneficial and is in process.

\subsection{Validation criteria}

Sole convergence of state estimation does not guarantee valid results and valid basecase for security functions.

A number of state estimation validation criteria may be introduced such as: cost value before and after bad data analysis, violation of estimated generators power limits, maximal percentage losses, observability level etc. 


\subsection{Integration with PMU measurements}

Serbian TSMO has furnished six phase measurement units (PMUs) and it has been planned to integrate them into state estimator application in the future.

Locations of these PMUs are carefully chosen and they will be installed on the most important $400 \mathrm{kV}$ tielines towards neighbouring systems, in Serbian substations.

WAMS measurements will have to be down sampled from $20 \mathrm{~ms}$ to $2 \mathrm{~s}$, which is a SCADA acquisition sampling rate in our SCADA system.

These measurements will be given the biggest accuracy and it is expected that they would make our state estimator more reliable in regard of a solution accuracy and convergence success.

\subsection{An open and flexible system}

Two SCADA/EMS systems working in parallel in Serbian TSMO have been running for years in production and one of those two systems is pretty closed, and the other one, which is currently being upgraded, is relatively open to changes, which is very important.

\section{Conclusion}

Power grids are in constant upgrade and reconstruction. All these changes must be reflected on SCADA/EMS system, which requires extensive manual entry and editing. This requires substantial engineering workforce. This is particularly the case in Serbian TSMO, where two different SCADA/EMS systems run in parallel. Lately, the number of substations covered with telemetry also substantially increased, which requires great model enlargement.

All the above proposed measures and features have a goal to increase data accuracy and consistency, resulting in more accurate state estimator solution and convergence reliability.

To make a state estimator functioning better in real time, a good cooperation between engineers is crucial. For Serbian system, aside from SCADA/EMS engineers, which must have a strong background in Power systems and IT, and who build and maintain SCADA/EMS model and check applications and servers continuously, it would be optimal to have one dispatcher and one Power system analysis engineer checking and tuning it on a daily basis.

Real time applications require shifts. Two persons would have to devote its full attention to its proper functioning and surveilling during one shift, and at least two SCADA/EMS engineers would have to cooperate with them to be able to do all the necessary modifications on a daily basis.
Since SCADA/EMS operates in real-time, there are continuous changes in the system, requiring even more automatization, where engineers would get alarm messages, containing already aggregated information, leading to the problem solutions quicker.

Developing new ideas and upgrading software and hardware, based on experience and new needs, investing in continuous engineering education and knowledge exchange with colleagues from other TSMOs is essential.

\section{References}

[1] A. Bose, K. A. Clements, "Real-Time Modeling of Power Networks", Proceedings of IEEE - Special Issue on Computers in Power System Operations, pp. 1607-1622, December 1987

[2] A. Monticelli, "STATE ESTIMATION IN ELECTRIC POWER SYSTEMS - A Generalized Approach", Kluwer's Power Electronics and Power Systems Series, Kluwer Academic Publishers, Norwell, Massachusetts, USA, 1999

[3] Trhulj J., Jakupovic G., Stojic M., Cukalevski N., "Real-Time Power System State Estimator within National Control Centre of Serbia", EUROCON 2005, November 22-24, 2005 Belgrade, Serbia

[4] Milos Stojic, Goran Jakupovic, Ninel Cukalevski, "The IT Architecture and Applications for the Transmission System Operator Back-up Control Center" - Invited paper, DEMSEE 2010, 5th International Conference on Deregulated Electricity Market issues in South-Eastern Europe, September 23-24, 2010 Sitia - Greece

[5] Milos Stojić, Goran Jakupović, Ninel Čukalevski, „One Implementation of Network Topology Processor Application for the TSO Back-up Control Center“,DEMSEE 2011 6th International Workshop on Deregulated Electricity Market Issues in SouthEastern Europe, 20.-21. September 2011, Bled, Slovenia

[6] Miloš Stojić, Goran Jakupović, Ninel Čukalevski, Jelena Veselinović, "New SCADA/EMS system in Serbian National Control centre", CG KO CIGRE, Montenegro, Igalo, 2015

[7] Jelena Veselinović, Boris Filipović, Implementation of the State Estimator in NDC Elektromreža Srbije", Juko CIGRE, Vrnjačka Banja 2007, Serbia 


\section{Biographies}

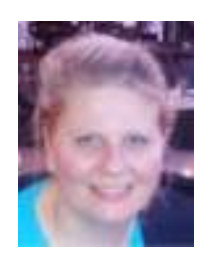

Jelena Veselinović was born in Sarajevo, Yugoslavia (now Bosnia and Herzegovina) in 1977. She has graduated and received her Dipl. Ing degree at University of Belgrade, School of Electrical Engineering, Power Systems Department. She spent a year working on a reaserch project related to HVDC links in multi-area systems at EPFL, Lausanne, Power Systems Department (Swiss Confederation Government Scholarship, 2008), performing dynamic simulations. She is with Serbian TSMO PE Elektromreža Srbije, Operational Technology Department, since 2006. Areas of her professional interest and expertise include SCADA/EMS/DTS, System Operation and Control, IT, Dynamic simulations. She is a member of study committee C2 (System Operation and Control) of Serbian National CIGRE Committee.

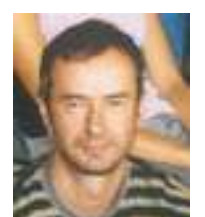

Miloš Stojić was born in Belgrade, Yugoslavia (now Serbia) in 1961. He has graduated and received his Dipl.Ing. at University of Belgrade, School of Electrical Engineering, Power Department in 1984. In 1992 he received Mr.Ing. in Systems and Logistics, University of Belgrade, Department for Systems and Logistics. Areas of his professional interest and expertise include SCADA/EMS software design and development, especially State Estimation including Network Topology. Other areas of interest include design and development of electronic devices for blind people. Since 1988 he is with Mihajlo Pupin Institute, Automation and Control department, Power Systems Control group. He is a member of study committee C2 (System Operation and Control) of Serbian National CIGRE Committee.

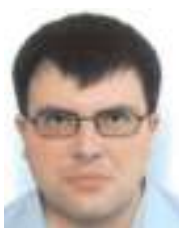

Goran Jakupović was born in Belgrade, Serbia on May $9^{\text {th }}$ 1971. He has graduated and received his Dipl.Ing. and Mr.Ing. degrees in Control Systems at Electronics, Communication and Control department of School of Electrical Engineering, University of Belgrade. Areas of his professional interest and expertise include: Control systems design, power system control applications, SCADA/EMS software technology and real-time control systems, applications of fuzzy logic and intelligent control in (power) control systems. He is with Mihajlo Pupin Institute Automation and Control department since 1995, first with intelligent systems group, and from 1996, with Power Systems Control group. $\mathrm{He}$ is a member of study committee C2 (System Operation and Control) of Serbian National CIGRE Committee. 